\title{
Metachronal waves in Cellular Automata: Cilia-like manipulation in actuator arrays
}

\author{
Ioannis Georgilas ${ }^{1,2}$, Andrew Adamatzky ${ }^{1,2}$, David Barr ${ }^{3}$, Piotr Dudek $^{3}$, and \\ Chris Melhuish ${ }^{2}$ \\ 1 International Centre for Unconventional Computing, University of the West of \\ England, Frenchay Campus, BS16 1QY, Bristol, UK \\ giannis.georgilas@uwe.ac.uk, \\ http://uncomp.uwe.ac.uk/giannis/Home.html \\ 2 Bristol Robotics Laboratory, University of Bristol and University of the West of \\ England, T Block, Frenchay Campus, BS16 1QY, Bristol, UK \\ http://brl.ac.uk \\ 3 School of Electrical \& Electronic Engineering, University of Manchester, \\ Sackville Street Building, M13 9PL, Manchester, UK \\ http://personalpages.manchester.ac.uk/staff/p.dudek/default.htm
}

\begin{abstract}
Paramecium is covered by cilia. It uses the cilia to swim and transport food particles to its mouth. The cilia are synchronised into a collective action by propagating membrane potential and mechanical properties of their underlying membrane and the liquid phase environment. The cilia inspired us to design and manufacture a hardware prototype of a massively parallel actuator array, emulated membrane potentials via a discrete excitable medium controller and mechanical properties based on vibrating motors. The discrete excitable medium is a two-dimensional array of finite automata, where each automaton, or a cell, updates its state depending on states of its closest neighbours. A local interaction between the automata lead to emergence of propagating patterns, waves and gliders. The excitable medium is interfaced with an array of actuators. Patterns travelling on an automaton array manifest patterns of actuation travelling along the array of actuators. In computer models and laboratory experiments with hardware prototypes we imitate transportation of food towards mouth pore of the Paramecium. The hardware actuator arrays proposed could in future replace simple manipulators in demanding micro-scale application.
\end{abstract}

Keywords: cellular automata, multi-agent systems, natural collaboration

\section{Introduction}

Multi-agent engineering systems are ubiquitous. Their control is challenging [9]. A specific category of multi-agent systems are the smart surfaces [4], manipulator arrays replacing traditional manipulators in industrial applications specifically at the micro-scale. 


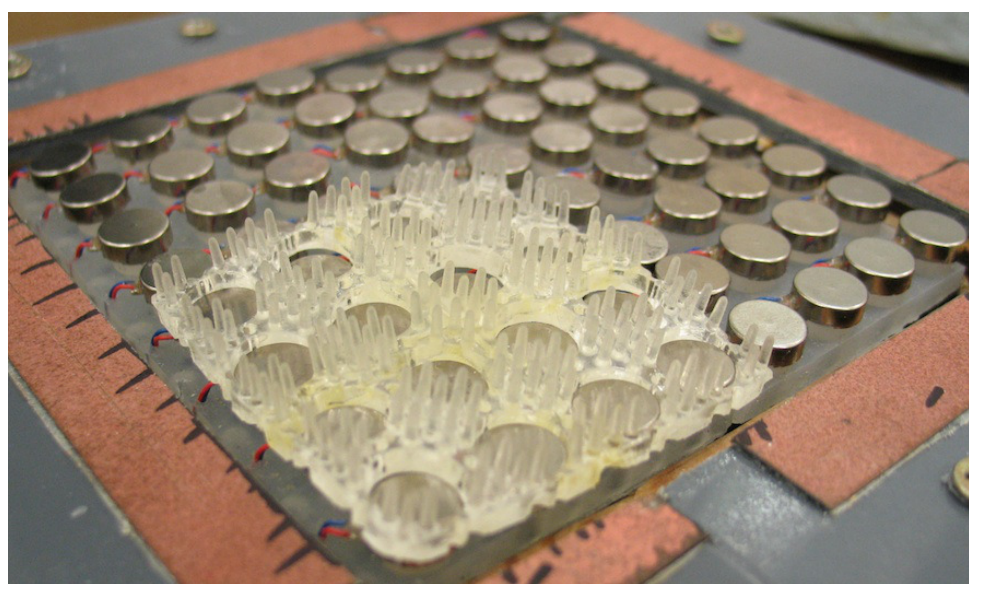

Fig. 1. Image of the bristles fabricated to emulate natural cilia. This design is used as an alternative to the membrane design described in [13]

Typical manipulation tasks in industrial environments are being performed by classic 6 -DOF manipulators. These robots are often very large, and can manipulate objects varying in size but are smaller than the manipulator itself. Typical arrangements of these manipulators are either as single units or in small groups, where collaborative tasks are achieved by properly planning the spatial trajectories of the individual units. Given the small number of agents involved the planning tasks can be solved analytical. Recently another class of manipulation tasks emerged: the micro-assembly or assembly on the molecular level. This novel field of manipulation leads to the creation of massive arrays of distributed small manipulators. The most common types are based on airjets [8], vibration based solutions [22] and micro electro-mechanical systems (MEMS) [10].

The actuators used by these systems are small, weak and inexpensive, but by using cooperative control, they are able to manipulate object much larger than their own size. Some types of cooperative control of smart surfaces has been analysed in [5, 18]. A manipulation based on cooperative behaviour in Cellular Automata (CA) has been studied in $[12,14]$. Presently we explore similarities of waves propagating in CA to biological cilia, like the ones found in Paramecium, and cilia controlling signalling [21] and how the latter allows the emergence of collaborative behaviour between the actuators of the prototype system developed in [13], and the bristled version as seen in Fig. 1. The artificial cilia of the hardware behave in a coordinated manner via excitation waves and propagating self-localised excitations that are travelling in the underlying CA lattice.

\section{Biological Coordination}

Coordination of multiple activities is amongst most intriguing mechanisms of natural systems [16]. It is still unclear how biological systems are coordinated to 


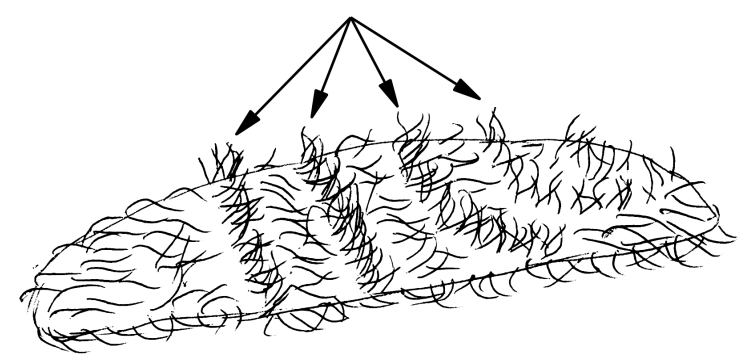

(a)

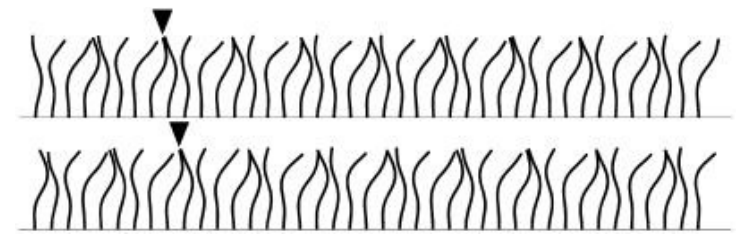

(b)

Fig. 2. (a) Image of Paramecium with cilia exhibiting metachronal waves (arrows), and (b) line of 40 cilia in the presence of a metachronal wave indicated by arrows [15].

produce specific behaviours. To answer this question the mechanics of spatialtemporal patterns have been investigated in different systems. The main idea is that chemical signalling mechanisms [7] are being used to produce these behaviours.

Of particular interest are the mechanisms that govern the operation of cilia. Cilia are organelle structures found in single-cell organisms used for propulsion, like in Paramecium Figs. 2(a), and manipulate particles of food, including other bacteria, towards the Paramecium's mouth pore. The latter is also the task by which the proposed artificial system will be evaluated. In multicellular organisms cilia are used as part of other systems, e.g. in the trachea for cleaning the incoming air from dust and small particles. The intriguing structure of a cilium has inspired building and control of artificial ones as flow generators. The fabrication technics involve, among other, magnetically actuated structures [11] and soft, electro-active polymer approaches [20].

The cilia coordination has been investigated in order to establish how the combined action of arrays of cilia produce effective fluidic flows that are propagating objects. The coordinated action of adjacent cilia and the break of symmetrical beating was analysed to establish the dynamics of macroscopic flow [15]. Also, phase-oscillator descriptions of the hydrodynamics interaction of beating cilia arrays have been formulated to allow the analytical investigation of cilia coordination [19]. The recurring subject of metachronal waves, Figs. 2(a), 2(b) and how this type of wave formation creates the maximum effective flow is analysed in $[2]$. 
Further to the signalling procedure that leads to the cooperative operation of cilia, the shape of the resulting flow, and related force vector, has influenced the current research. It has been proposed and demonstrated in modelling, that the $3 \mathrm{D}$ fluidic flow around an actuated cilium has the shape of a $3 \mathrm{D}$ vortex [6]. The $2 \mathrm{D}$ component of this motion generates the common shape of vortex with the vectors of acting forces rotating around a centre and converging to the generative specific spot, i.e. the cilium. The latter is the inspiration for the model that is used for describing the hardware behaviour of the system in [13].

\section{Cooperative smart surface}

\subsection{Cellular Automata}

It has been proposed that reaction-diffusion (RD) models can provide a framework to analyse signal propagation in biological systems [17]. The intrinsic simplicity of the RD approach facilitates simulation of the chemical and fluid dynamics of the cilia signalling methods that lead to their cooperative functionality. Based on this observation, CA as a form of modelling RD, are being used in this research to model the signals used for cilia coordination. CA are a type of distributed embedded controller that, via the state changing rules patterns emerge demonstrating collaborative performance. The investigation of the emerging patterns in CA have been extensively investigated for their information processing abilities [1].

In previous work we used the $2^{+}$-medium ruleset as the controller of the smart surface $[12,14]$. In brief, the neighbourhood of this ruleset is $3 \times 3$ cells and the rules are as follows; each cell, $x$ of a $2 \mathrm{D}$ lattice $\mathbf{L}$, can take three states, resting $(\cdot)$, excited $(+)$ and refractory $(-)$, for this manuscript whenever a $\mathrm{CA}$ lattice is depicted in a figure resting state will be black, excited state will be grey and refractory state will be white. A resting cell is excited if the number of excited neighbours is exactly two. An excited cell takes refractory state and a refractory cell takes a resting state unconditionally:

$$
x^{t+1}= \begin{cases}+, & x^{t}=\cdot \text { and } \sum_{y \in u(x)} \chi(y,+)=2 \\ -, & x^{t}=+ \\ \cdot, & \text { otherwise }\end{cases}
$$

where $u(x)=\{y \neq x \in \mathbf{L}:|x-y| \leq 1\}$ and $\chi(y,+)=1$ if $y=+$ and 0 , otherwise.

In this ruleset self-localisations, or wave-fragments, having an excited front, two cells being in the excited state ( + or grey in the pictures), and a refractory tail, two cells being in the refractory state (- or white in the pictures), travel in straight lines. These excitation waves can be considered analogs of metachronal waves that are traveling in the lattice. As shown in Fig. 3, ten wave-fragments traveling in a line are behaving in a similar manner to the waves in cilia of Fig. 2(b). 


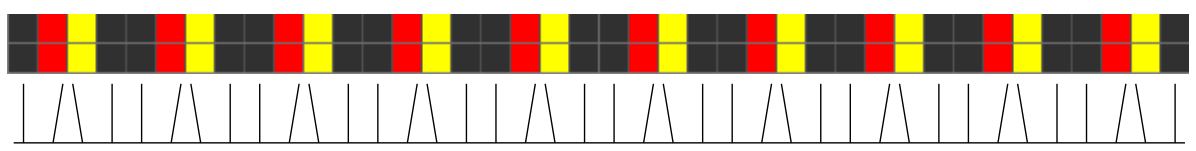

Fig. 3. Excitation waves travelling in a $40 \times 2$ cylindrical lattice. The 'equivalent' cilia metachonal wave is depicted below the CA states (grey is excited, white is refractory and black is resting).

Under the CA states is a side view of the 'equivalent' cilia. Each cilium can take central, left or right position. Although the beating pattern, the bending of the cilium, is simpler that in nature, the intended behaviour is emerging from the phenomenology of the wave propagation.

\subsection{Actuators and coupling with CA}

In order to achieve the vortex generation method of the cilia in 2D, we use vibrating motors interconnected using a flexible membrane. Each motor can rotate in both directions, clockwise and counter-clockwise. This directional operation modes, plus being off are mapped with the three CA states. Hence resting state $(\cdot)$ is mapped as motor off, excited $(+)$ is mapped as motor $C W$ and refractory $(-)$ is mapped as motor $C C W$, The motors are designed to vibrate radially, but being positioned on a flexible platform allows them to vibrate slightly in the axial direction. A more detailed description of the hardware can be found in [13].

The combination of the axial and radial vibrations create the vortex shaped force field on Fig. 5(a). This is similar to the 2D components of the vortexes generated by cilia bitting in fluids [6]. The generated force field is created by the rotation of a single motor. With arrows are the vectors of the force in specific position on the top of the membrane. The length of the vector represents the magnitude of the force at the point. As it is expected, the closer a vector is to the motor the higher the exerted forces are. If the forces are negligible no vector is shown (blank space in the figures).

In order to achieve controllable collaborative behaviour the CA states and the motor commands need to be mapped. This connection is straightforward since the three states of the CA can be connected with the three states of the motor. The resting state is connected with the motor being off, while excited and refractory are arbitrarily selected as motor rotating clockwise (CW) and motor rotating counter-clockwise $(\mathrm{CCW})$ respectively.

\section{Actuator Cooperative Behaviour}

To demonstrate similarities between manipulation methods in Paramecium and our actuator array we chosen a task of transporting 'food' object to a dedicated site of a manipulator, which imitates a 'mouth pore'. 


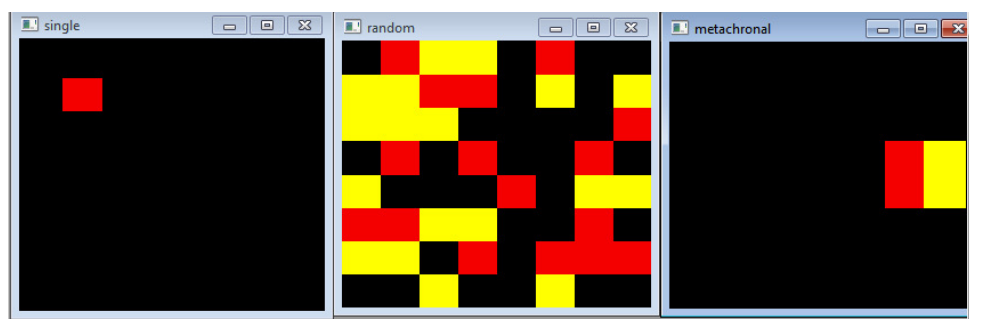

Fig. 4. Screenshot from the APRON environment where the control signals for the motors lattice are depicted in colour matrices. Single motor, random signal and metachronal wave (at frame 1).

We compare three different control actuation signals, single motor, random and metachronal waves in CA. The motor commands were generated using the software APRON and the physical behaviour was simulated in MATLAB. The location of the object is selected randomly and in the lower right corner of the lattice. Since the model is deterministic a single simulation is needed for each of the three signalling methods.

APRON. Array Processing enviRONment [3] is used as the control signals development and simulation environment. It is a real-time, interactive and highly visual simulation platform for working with and debugging $2 \mathrm{D}$ arrays of data and rapidly prototyping array based algorithms. APRON scripts treat arrays as a primitive data type, where the user sequentially defines operations between them, and computation is performed in an element-wise manner. Individual elements can be disabled based upon both global and local data and conditions, yielding local autonomy and algorithmic branching with the array. The CA rules were developed in open-loop and a frame of the output from each signalling method is shown Fig. 4.

MATLAB The information from APRON was then transferred to MATLAB where the generation of the vortex force-field for each motor was generated. To create the total force-field of a single motor a combination of coupled oscillators, (2a) and membrane wave oscillations (2b) was used. The former provided the components for the behaviour of each motor as affected by the rotation of the adjacent motors, while the latter describes the dynamics of the membrane that covers all the motors and connects them.

$$
\begin{gathered}
F_{C}(t)-k d-p \dot{d}=m \ddot{d} \\
\frac{\partial^{2} z}{\partial x^{2}}+\frac{\partial^{2} z}{\partial y^{2}}=\frac{1}{c^{2}}\left(\frac{\partial^{2} z}{\partial t^{2}}+p \frac{\partial z}{\partial t}\right), c^{2}=F_{W} / \rho
\end{gathered}
$$

where, $F_{C}(t)$ is the actuation force (motor's torque), $k$ and $p$ are the spring and damping coefficients of the membrane, $m$ is the mass of the eccentric weight 


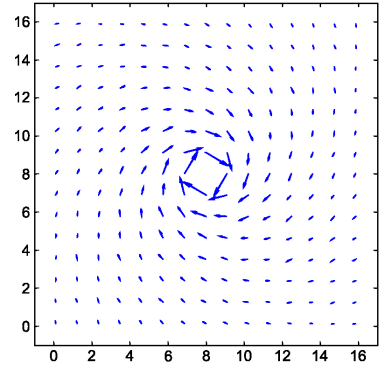

(a)

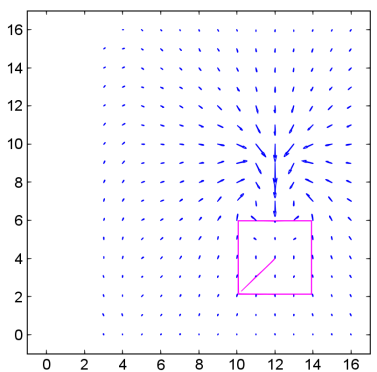

(d)

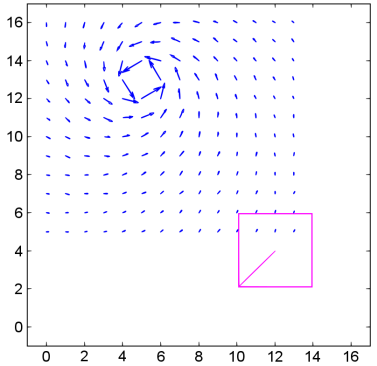

(b)

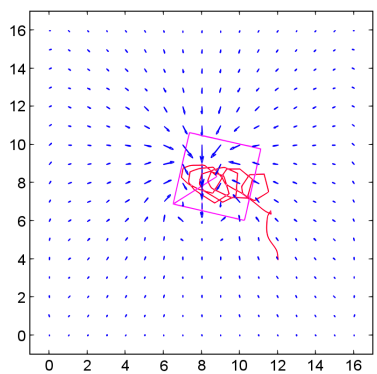

(e)

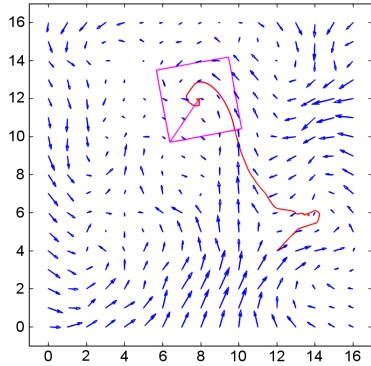

(c)

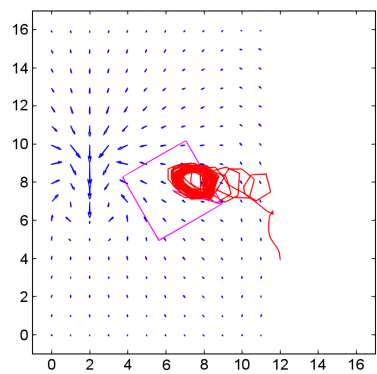

(f)

Fig. 5. Simulation frames from MATLAB with APRON generated control signals. The vectors of the force field, the rectangular object with a rotation indication line and the trajectory are depicted. (a) Is the vortex force-field created by a single motor, (b) is the single motor force field 'pulling' the object towards the centre of the lattice (frame $60),(c)$ is the trajectory of the object under a randomly generated force field (frame 400), (d)-(f) are frames 0, 60 and 400 of the trajectory under the metachronal wave signal. Object is placed at coordinates $(12,4)$. Axis are simulation based units.

and $d, \dot{d}$ and $\ddot{d}$ is the displacement, velocity and acceleration along the axis of oscillation. And $c$ is a speed of wave travelling on the membrane, $F_{W}$ is a force generated by the wave and $\rho$ is a density of the membrane's medium. The membrane is considered homogeneous and isotropic. The term $p \dot{d}=p \frac{\partial z}{\partial t}$ is again the damping effect of the membrane as in (2a) but in the $z$ axis.

The combined force-field was then used to calculate the displacement and rotation of the object using the equations for normally accelerated motion (3).

$$
\begin{array}{r}
x(t)=x(t-\Delta t)+u_{x}(t-\Delta t) \cdot \Delta t+\frac{\mathrm{a}_{x}(t-\Delta t) \cdot \Delta t^{2}}{2} \\
y(t)=y(t-\Delta t)+u_{y}(t-\Delta t) \cdot \Delta t+\frac{\mathrm{a}_{y}(t-\Delta t) \cdot \Delta t^{2}}{2} \\
r(t)=r(t-\Delta t)+\omega(t-\Delta t) \cdot \Delta t+\frac{\alpha(t-\Delta t) \cdot \Delta t^{2}}{2}
\end{array}
$$




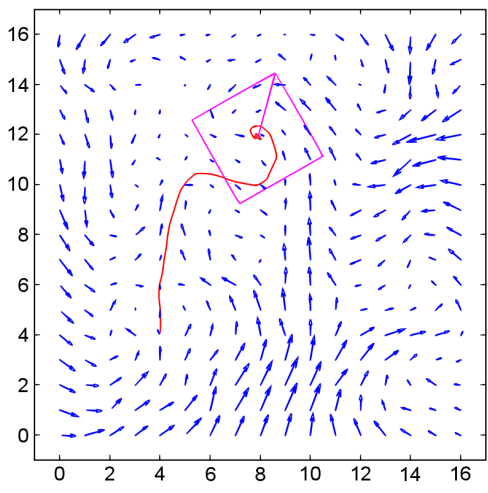

(a)

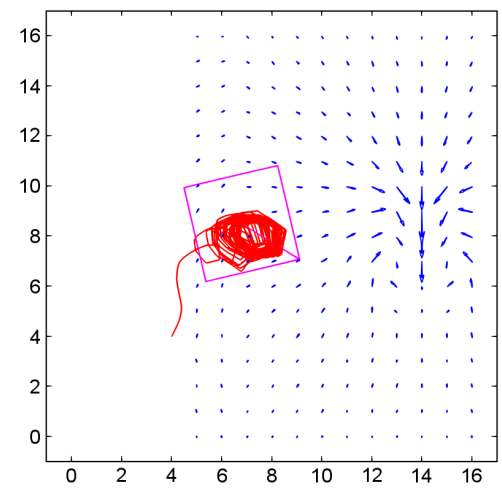

(b)

Fig. 6. Object trajectory for random and CA metachronal wave control signal. Initially the object is placed in coordinates $(4,4)$ in this experiment

where $x(t), y(t), r(t)$ are the position and rotation of the object at time $t, u_{x}(t)$ and $u_{y}(t)$ are the linear velocity in the two directions and $\omega(t)$ is the angular velocity, and finally, $\mathrm{a}_{x}(t), \mathrm{a}_{y}(t)$ are the linear and $\alpha(t)$ is the angular acceleration respectively, as calculated by $F_{M}=m \cdot$ a and $M_{M}=\alpha(t) \cdot I_{d}$, where $F_{M}$ is the resultant force of $F_{C}$ and $F_{W}, M_{M}$ the rotational momentum of this force, and $I_{d}$ the inertia and $m$ the mass of the object. All calculations are performed in specified simulation time intervals, $\Delta t$.

The three different control signals are representing three methods for achieving the task. The first is to enable a single motor (cilium) symmetrically from the location of the object and try to 'pull' the 'food' towards the 'mouth pore', Fig. 5(b). The second is a randomly generated control signal, Fig. 5(c), where the 'cilia' are flapping randomly to try to move the 'food' towards the 'mouth pore'. Finally, the third control signal is an excitation wave traveling horizontally on the lattice. The lattice is assumed being a torus and as such the wave is 'wrapping' around from left to right, Fig. 5(d)-5(f).

\subsection{Discussion}

Analysing the trajectories from the different control signals we can deduce some interesting results regarding the cooperative attributes of the various control signalling.

The single motor approach, with the motor trying to 'pull' the object towards the centre fails even to start moving the object. Investigating the exhibited forces we find that the single motor is not able to exert the friction between the object and the membrane. This is a demonstration that a single 'cilium' fails to achieve the task and some form of cooperative action needs to take place. 
The random motor approach overcome the lack of power, since it does move the object from its initial position. Nonetheless, the object is moving in a random path. Furthermore, it might be locked in local attractors that will not coincide with the intended target. Hence, the use of multiple 'cilia' is necessary to produce manipulation, but random 'beating', hence signalling, does not create controllable behaviour.

Finally, the CA excitable/metachronal wave both moves the object and reaches the intended target. The synchronised movement of the motors create the collaborative behaviour that natural cilia create. Hence, this type of control signalling can create controllable collaborative behaviour.

In order to demonstrate the generalisation of the proposed method, in Fig. 6 the trajectory paths of the object from a different location are depicted. As can be easily noted, the random force-field, Fig. 6(a), exhibits the same random/local attractor behaviour, while the CA metachronal wave, Fig. 6(b), moves the object towards the target.

\section{Conclusions}

Coordination is crucial for the function of biological systems. There is a great deal of research being done in order to unravel the process with which systems of multiple agents are communicating and collaborating, like the case of cilia in the Paramecium. One of the most prominent explanations is the use of metachronal waves to co-ordinate the ciliate movements.

With this work we demonstrated a method to replicate the metachronal waves of cilia utilising reaction-diffusion models of CA. We demonstrated how excitation waves travelling in the CA lattice can phenomenologically model waves in cilia arrays and how this waves can be used as signals to control a prototype actuators array, a smart surface.

Using these metachronal waves in the CA medium the actuators exhibited a controlled collaborative behaviour. The ability to succeed in a simple manipulation task, has been demonstrated through a set of experiments. The metachronal wave control signals were compared with single actuator activation, lacking sufficient force, and random control signalling, lacking controllable manipulation.

Future work could involve developing machine learning and evolutionary computing approaches to investigate the possible collaborative behaviour of the system. Hence, the solutions to the optimal cooperation method can emerge by the analysis of the potential solution space. Furthermore, a consistent understanding of the dynamics of the cooperative behaviour must be investigated. This will be achieved by developing further the vortex modelling for the actuators. Also, a connection of the modelled behaviour with the hardware described in [13] will prove the suitability of the approach under real-world conditions. Furthermore, the potential of the proposed method to be used as a generic controller for other system should be investigated. Especially as a solution for scalability issues in multi-agent systems, since the metachronal CA-generated wave can inherently scale without increasing the complexity of the underlying hardware. 


\section{Bibliography}

[1] Adamatzky A (2001) Computing in non-linear media and automata collectives. Institute of Physics Publishing

[2] Aiello E, Sleigh MA (1972) The metachronal wave of lateral cilia of mytilus edulis. The Journal of cell biology 54(3):493-506

[3] Barr DR, Dudek P (2009) Apron: A cellular processor array simulation and hardware design tool. EURASIP Journal on Advances in Signal Processing 2009:9

[4] Bohringer KF, Donald BR, Mihailovich R, MacDonald NC (1994) Sensorless manipulation using massively parallel microfabricated actuator arrays. In: Robotics and Automation, 1994. Proceedings., 1994 IEEE International Conference on, IEEE, pp 826-833

[5] Bohringer KF, Bhatt V, Donald B, Goldberg K (2000) Algorithms for sensorless manipulation using a vibrating surface. Algorithmica (New York) 26(3-4):389-429

[6] Chen D, Norris D, Ventikos Y (2009) The active and passive ciliary motion in the embryo node: A computational fluid dynamics model. Journal of Biomechanics 42(3):210 - 216, DOI 10.1016/j.jbiomech.2008.10.040, URL http://www.sciencedirect.com/science/article/pii/S0021929008005472

[7] Christensen ST, Pedersen LB, Schneider L, Satir P (2007) Sensory cilia and integration of signal transduction in human health and disease. Traffic $8(2): 97-109$

[8] Delettre A, Laurent G, Le Fort-Piat N (2011) 2-dof contactless distributed manipulation using superposition of induced air flows. In: 2011 IEEE/RSJ Int. Conf. Intelligent Robots and Systems, IROS'11, San Francisco, CA, pp 5121-5126, DOI 10.1109/IROS.2011.6048251

[9] Ferber J (1999) Multi-agent systems: an introduction to distributed artificial intelligence, vol 1. Addison-Wesley Reading

[10] Fujita H, Ataka M (2010) System configuration and fabrication technology for distributed mems. In: 1st Worksh. Hardw. Softw. Impl. Contr. Distr. MEMS, dMEMS'10, Besancon, pp 1-5

[11] Gauger EM, Downton MT, Stark H (2009) Fluid transport at low reynolds number with magnetically actuated artificial cilia. The European Physical Journal E 28(2):231-242

[12] Georgilas I, Adamatzky A, Melhuish C (2012) Manipulating objects with gliders in cellular automata. In: 2012 IEEE International Conference on Automation Science and Engineering (CASE), IEEE, pp 936-941

[13] Georgilas I, Adamatzky A, Melhuish C (2012) Towards an intelligent distributed conveyor. Advances in Autonomous Robotics pp 457-458

[14] Georgilas I, Adamatzky A, Melhuish C (2013) Manipulating with excitations: Waves or gliders? In: Workshop notes of the ICRA Workshop in Unconventional Approaches to Robotics, Automation and Control Inspired by 
Nature, Karlruhe, vol International Conference in Robotics and Automation (ICRA)

[15] Guirao B, Joanny JF (2007) Spontaneous creation of macroscopic flow and metachronal waves in an array of cilia. Biophysical journal 92(6):1900-1917

[16] Kelso J, Schöner G (1987) Toward a physical (synergetic) theory of biological coordination. In: Lasers and synergetics, Springer, pp 224-237

[17] Kondo S, Miura T (2010) Reaction-diffusion model as a framework for understanding biological pattern formation. Science 329(5999):1616-1620

[18] Konishi S, Fujita H (1995) System design for cooperative control of arrayed microactuators. In: Micro Electro Mechanical Systems, 1995, MEMS'95, Proceedings. IEEE, IEEE, p 322

[19] Lenz P, Ryskin A (2006) Collective effects in ciliar arrays. Physical biology $3(4): 285$

[20] Sareh S, Rossiter J, Conn A, Drescher K, Goldstein RE (2012) Swimming like algae: biomimetic soft artificial cilia. Journal of The Royal Society Interface 10(78)

[21] Satir P, Christensen ST (2008) Structure and function of mammalian cilia. Histochemistry and cell biology 129(6):687-693

[22] Vose T, Umbanhowar P, Lynch K (2010) Toward the set of frictional velocity fields generable by 6-degree-of-freedom oscillatory motion of a rigid plate. In: 2010 IEEE Int. Conf. Robot, ICRA '10, Anchorage, AK, pp 540-547 